\author{
Revista de Psicología Vol. 34 (1), 2016 (ISSN 0254-9247) \\ http://dx.doi.org/10.18800/psico.201601.001
}

\title{
Doble excepcionalidad: análisis exploratorio de experiencias $y$ autoimagen en estudiantes chilenos ${ }^{1}$
}

\author{
María Paz Gómez Arizaga ${ }^{2}$, Maria Leonor Conejeros-Solar ${ }^{3}$, \\ Katia Sandoval Rodríguez y Solange Armijo Solís ${ }^{5}$, \\ Universidad de Los Andes, y Pontificia Universidad Católica \\ de Valparaiso, Chile
}

\begin{abstract}
El presente estudio explora la construcción de autoimagen y el tipo de experiencias ocurridas con compańeros y profesores en la condición de doble excepcionalidad. Se analizan cuatro casos de estudiantes de 8 a 15 años que presentaban trastorno de déficit de la atención (TDAH) con alta capacidad y trastorno del espectro autista (TEA). Los resultados indican que los estudiantes presentaban una noción de la discrepancia de su condición a pesar de desconocerla; estaban motivados al aprendizaje, pero se aburrían con tareas poco desafiantes o repetitivas; presentaban buena relación con profesores y compańeros, sin embargo, para el grupo de estudiantes con alta capacidad y TEA existía una connotación negativa. Se discuten implicancias para la investigación y práctica educativa.

Palabras clave: doble excepcionalidad, alta capacidad, trastorno de déficit de la atención, trastorno del espectro autista, contexto escolar.
\end{abstract}

1 Las autoras agradecen el apoyo de FONDECYT Proyecto $\mathrm{N}^{\circ} 1151030$.

2 Doctora en Educación Especial por la Universidad de Arizona, Estados Unidos y profesora investigadora en la Facultad de Educación de la Universidad de los Andes. Dirección postal: Álvaro del Portillo 12.455, Las condes, Santiago, Chile. Contacto: mpgomez@uandes.cl

3 Doctora en Educación por la Universidad de Concepción, Chile y profesora adjunta de la Escuela de Pedagogía, Carrera de Educación Especial de la Pontificia Universidad Católica de Valparaíso. Dirección postal: Avenida El Bosque 1290, Campus María Teresa Brown de Ariztía, Viña del Mar, Chile. Contacto: leonor.conejeros@ucv.cl

4 Doctora en Ciencias de la Educación por Universidad de Granada, España y docente de la Carrera de Educación Especial en la Pontificia Universidad Católica de Valparaíso. Dirección Postal: Avenida El Bosque 1290. Viña del Mar, Chile. Contacto: katia.sandoval@ucv.cl

5 Licenciada en Educación Diferencial por la Pontificia Universidad Católica de Valparaíso. Dirección postal: Avenida Valparaíso 230, Departamento 404, Viña del Mar, Chile. Contacto: solange.armijo.solis@gmail.com 


\section{Dual exceptionalities: Exploratory analysis of experiences and self-image in Chilean students}

This research explores the manifestation of dual exceptionality, regarding self-image and the experiences students have had with classmates and teachers. The sample was comprised of four students ages 8 to 15 that exhibited ADHD with giftedness and ASD with giftedness. In-depth interviews were conducted with the students. Results showed that students who had a notion of their discrepancies were motivated towards learning, but became bored when tasks were not challenging. They also had good relationships with their teachers and peers. However, meaningful social interactions had a negative connotation for the gifted/ ASD group. Implications for research and practice are discussed further.

Keywords: dual exceptional, high ability, attention deficit disorder, autism spectrum disorder, school context.

\section{Dupla excepcionalidade: análise exploratória das experiências e autoimagem em estu- dantes chilenos}

Esta pesquisa explora a manifestaçáo da dupla excepcionalidade referente à construção da autoimagem e o tipo de experiências com colegas e professores. Os casos de quatro (4) estudantes de 8 a 15 anos que apresentavam transtorno do déficit de atenção (TDAH) com alta capacidade e transtornos do espectro autista (TEA) com alta capacidade, foram analisados. Os resultados indicam que os estudantes tinham uma noção da discrepância de sua condição, apesar de ignorá-la. Eles estavam motivados a aprender, mas ficavam entediados com tarefas pouco desafiantes ou repetitivas. Além disso, apresentavam boas relaçóes com colegas e professores, no entanto, para o grupo de alunos com TEA e alta capacidade, esta relação tinha uma conotação negativa. As implicaçóes para a pesquisa e a prática educativa, são discutidas.

Palavras chave: dupla excepcionalidade, alta capacidade, transtorno do déficit de atenção (TDAH), transtornos do espectro autista (TEA), contexto escolar. 
Quizás uno de los aspectos más desafiantes de este campo de investigación ha sido contar con una definición clara y operacional de la doble excepcionalidad. Afortunadamente, diversos estudios realizados en la última década a nivel internacional han posibilitado dilucidar el concepto para poder comprenderlo desde diferentes ámbitos: personal, social, familiar y escolar.

$\mathrm{Si}$ se define de manera general, la doble excepcionalidad puede ser entendida como la posesión simultánea de un alto potencial y una discapacidad (Assouline \& Whiteman, 2011; Foley Nicpon, Allmon, Sieck \& Stinton, 2011; Dixon \& Moon, 2006; Baum \& Owen, 2004; Neihart, 2000). Esta paradoja se conforma entonces a partir de la combinación de una alta capacidad en áreas específicas (p. e. área intelectual, artística, etc.) con dificultades de aprendizaje, en la atención, déficits sensoriales, desórdenes emocionales graves, deficiencias motrices, y deficiencias cognitivas (Pardo de Santayana, 2002) que impiden su adecuado desarrollo.

Una de las complejidades de este fenómeno es que una excepcionalidad generalmente puede llegar a enmascarar a la otra, lo cual puede resultar en que los estudiantes queden sin recibir adecuada atención (McCoach, Kehle, Bray \& Siegle, 2001). Es en este sentido que, a nivel escolar, los estudiantes pueden ser identificados por una sola excepcionalidad y, por lo tanto, solo una de las necesidades es atendida a través de servicios especiales, tales como programas de atención al talento o servicios orientados a dar respuesta a la necesidad de apoyo derivada de su discapacidad o trastorno. En el caso de Chile, lo segundo es lo más probable que ocurra, dado el incremento en la atención a la discapacidad en los últimos ańos y el aumento de personal calificado en los establecimientos educacionales, a través de los Programas de Integración Escolar (PIE), en donde evalúan y/o brindan atención especializada a través de grupos de integración (Tenorio, 2011). 
La discapacidad concomitante con el talento puede ser diferente dependiendo de cómo se manifieste (ej. cognitiva, emocional, motriz, etc.). Para esta investigación, el foco estará en el Trastorno por Déficit de Atención (TDAH) y el Trastorno del Espectro Autista (TEA), por las siguientes razones: (a) la alta prevalencia de estos dos trastornos en los programas de integración escolar, especialmente de TDAH (MINEDUC, 2012), (b) la falta de investigación a nivel internacional en relación con la doble excepcionalidad con TEA (Rubenstein, Schelling, Wilczynski \& Hooks, 2015) y (c) la necesidad de establecer un punto de partida en la investigación de doble excepcionalidad en Chile/Latinoamérica.

\section{Alta capacidad}

En la literatura se pueden encontrar diversas definiciones y conceptualizaciones de la alta capacidad, las cuales se desprenden de diferentes paradigmas, tales como enfoques orientados a la psicometría y otros hacia el contexto social. El grado de acuerdo que parece surgir desde los investigadores, es que el constructo de la alta capacidad es complejo y multifactorial e incluye capacidades y desempeños en áreas tanto cognitivas como no cognitivas (Heller, Mönks, Sternberg \& Subotnik, 2000). En Chile no se cuenta con una definición por parte de organismos gubernamentales.

Sin embargo, los actuales programas de enriquecimiento para estudiantes talentosos que existen en el país, tales como PENTA UC (2015), BETA PUCV (2015), PROENTA (2015), UFRO, entre otros, han adoptado el mismo enfoque hacia la alta capacidad, denominado Modelo Diferenciado de Dotación y Talento (MDDT) de Francoys Gagné (2011) en su versión 2.0. Una de las principales razones por las que se ha adoptado este modelo en Chile es debido a su conceptualización flexible y equitativa en cuanto a la alta capacidad: mientras que algunos sitúan su prevalencia entre un $2 \%$ a $5 \%$ de la población, el MDDT lo hace en el 10\%. La dotación (o potencial) en este modelo 
es entendida como la posesión y uso de habilidades superiores expresadas en al menos un dominio (intelectual, creativo, social, perceptual y físico), el cual sitúa a la persona en el $10 \%$ superior de su grupo.

El talento, por otra parte, se define como el manejo superior de habilidades que han sido desarrolladas sistemáticamente y que sitúan a la persona en el $10 \%$ de aquellos que se encuentran trabajando en esos dominios. Un elemento crítico para el proceso de desarrollo de potencial a talento es la presencia de catalizadores que pueden favorecer $\mathrm{u}$ obstaculizar este proceso (Gagné, 2011).

\section{Trastorno por déficit de atención}

El TDAH es uno de los desórdenes de la infancia más comunes, el cual está asociado a ciertos tipos de manifestaciones como inatención, impulsividad y/o hiperactividad (Rinn \& Reynolds, 2012). La co-ocurrencia de TDAH y alta capacidad es muy compleja tanto para la identificación como la intervención, debido a las características superpuestas que ambas pueden presentar (Baum, Olenchak \& Owen, 1998) y los diagnósticos erróneos que muchas veces ocurren en estudiantes con alta capacidad, en donde estos son etiquetados como TDAH sin serlo. Otra característica de este tipo de doble excepcionalidad es que habitualmente la alta capacidad puede enmascarar el TDAH, pero al mismo tiempo la manifestación del TDAH se puede traducir en un pobre desempeño académico y conductas disruptivas a nivel escolar, un escenario que lleva a un escepticismo por parte de los docentes acerca de la presencia de una alta capacidad en el estudiante (Neihart, 2003).

Sin embargo, algunos investigadores han encontrado rasgos únicos en este grupo en particular, tales como la baja persistencia en tareas irrelevantes, un alto nivel de actividad y el cuestionamiento de las reglas existentes (Webb \& Latimer, 1993). Otras características son una tendencia a la soledad, aburrimiento, autocrítica permanente, sensibilidad emocional, dominación y sentimientos de rechazo hacia figuras de autoridad, menos atención al detalle y dificultad para cambiar 
de tarea (Flint, 2001). En cuanto a esto último, la discusión se ha centrado en el hecho que los estudiantes son capaces de focalizarse por períodos más largos de tiempo y persistir más allá del error en ciertas tareas que son desafiantes en comparación a un estudiante que presenta únicamente TDAH (Pardo de Santayana, 2002). Otros investigadores se refieren a este estado como "hiperfocus", es decir, un foco extremo en un estímulo (Hua, Shore \& Makarova, 2012; Kaufmann, Kalbfleisch \& Castellanos, 2000), que también ha sido conceptualizado como un "interés obsesivo". Este hiperfoco puede ser desafiante, especialmente dentro de un sistema escolar que puede llegar a ser muy rígido o estructurado.

\section{Trastorno del espectro autista}

El Manual Diagnóstico y Estadístico de los Desórdenes Mentales (DSM-5), en su quinta versión, entrega una concepción más amplia del autismo, introduciendo el concepto de espectro en vez de desórdenes separados. Otro criterio importante incluido en el DSM-5 ha sido que, para fines diagnósticos, el niño debe haber presentado síntomas dentro de los 36 primeros meses de edad. Dentro de este continuum, los individuos con TEA pueden presentar síntomas con distinto grado de severidad en áreas tales como la comunicación (ej. mostrar respuestas inapropiadas en contextos sociales, baja comprensión del lenguaje no verbal) y conducta (ej. rutinas rígidas, sensibilidad a los cambios) (American Psychiatric Association, 2013).

Las creencias iniciales en cuanto a los alumnos con alta capacidad y TEA eran que estos tenían las mismas dificultades de razonamiento social que sus pares con TEA no talentosos. Sin embargo, estudios más recientes sugieren que los estudiantes con doble excepcionalidad eran capaces de adaptarse mejor debido a su comprensión de las interacciones sociales, lo cual puede ser explicado por su inteligencia verbal (Lovecky, 2004). No obstante, otros autores han reportado que este grupo sigue exhibiendo conductas como escasa comprensión de reglas y directrices de comportamiento social, así como conductas sociales atípicas (Neihart, 2000). 


\section{Contexto escolar y doble excepcionalidad}

\section{Aprendizaje}

Las experiencias escolares de los estudiantes doblemente excepcionales pueden estar influenciadas por distintos factores, pero una de las temáticas que han sido descritas frecuentemente en la literatura establece una relación con la discrepancia entre las habilidades de los estudiantes y la discapacidad que presentan, la cual no siempre ha sido identificada y es confundida con bajo rendimiento, baja motivación, "flojera", y también porque la alta capacidad tiende a compensar el déficit y por lo tanto las debilidades concomitantes pueden no presentarse tan claramente. Algunas dificultades que pueden obstaculizar el proceso de aprendizaje de un estudiante con doble excepcionalidad pueden ser auditivas, visuales, de procesamiento secuencial, funcionamiento ejecutivo, velocidad de procesamiento, y recuperación de información (Trail, 2006).

De modo general, los estudiantes doblemente excepcionales son percibidos por otros miembros de la comunidad escolar de manera diferente a la visión tradicional de la alta capacidad. Se les ve manifestando conductas contradictorias de aprendizaje, ya que en ocasiones demuestran un alto nivel de habilidades como resolución de problemas y creatividad frente a ciertas tareas, y en otras situaciones presentan un bajo desempeño en diferentes actividades, lo cual se traduce en un rendimiento descendido en una o más áreas académicas (Winebrenner, 2003).

En cuanto a sus preferencias de aprendizaje, los estudiantes eligen actividades prácticas y/o demostraciones en donde puedan tener una aproximación de carácter inductivo en su proceso de aprendizaje (Silverman, 2003; Van Tassel-Baska, Feng, Swanson, Quek \& Chandler, 2009; Willard-Holt, 1998; Winebrenner, 2003). Asimismo, como cualquier otro estudiante con alta capacidad, pueden aburrirse con actividades poco motivantes o estimulantes, cuando lo planificado no coincide con sus intereses, o cuando el currículum avanza a un ritmo lento (Lubinski, 2004; Pereles, Omdal \& Baldwin, 2009). 
Un rasgo distintivo que ha sido mencionado en la literatura es una sensación permanente de fracaso a nivel escolar por no ser capaces de realizar actividades simples como leer o escribir (Silverman, 2003). Sus conductas académicas se relacionan con lo que les sucede a aquellos estudiantes no talentosos que han sido diagnosticados con una dificultad de aprendizaje: resistencia a trabajar, conductas disruptivas en el aula, distractibilidad, desvalorización de su establecimiento escolar, y bajo desempeño académico (Neihart, 2008).

A pesar que los estudiantes con doble excepcionalidad tienden a reportar sentimientos de baja eficacia en cuanto a su desempeño escolar, algunos investigadores han encontrado factores protectores (Dole, 2000) que pueden ayudar a compensar las malas experiencias escolares, como la persistencia, la humildad, la conciencia de sí mismos, y los intereses personales (Silverman, 2000). De igual manera, otro aspecto positivo es que los estudiantes tienden a usar sus fortalezas para compensar los problemas que puedan aparecer. Willard-Holt, Weber, Morrison y Horgan (2013) encontraron que esta compensación era realizada a través de estrategias de resolución de problemas, y que los estudiantes las implementaban en diferentes contextos; sin embargo, en este estudio, los autores no pudieron dilucidar el proceso de adquisición de dichas estrategias que muchas veces operan de forma inconsciente.

En cuanto a las experiencias de aprendizaje de estudiantes con TDAH y alta capacidad, Assouline, Foley Nicpon y Huber (2006) reportaron vivencias negativas de estos estudiantes en los establecimientos escolares. En el estudio de casos realizado por estos autores, encontraron que uno de los estudiantes se aburría y distraía fácilmente con actividades monótonas como la escritura. Al mismo tiempo, este joven sentía que no había preocupación por su desarrollo educativo y bienestar. Otro participante de la investigación se sentía poco motivado y etiquetado por otros como "flojo".

En cuanto a estudiantes con alto potencial y TEA, Rubinstein y colaboradores (2015), en un estudio cualitativo acerca de las experiencias escolares y familiares de alumnos con TEA, encontraron que, en términos de aprendizaje, había una tendencia hacia una combinación 
de alto potencial con intereses "intensos", es decir, los estudiantes manifestaban sus capacidades a través de un foco intensificado en ciertos temas, que iban desde dinosaurios hasta computadores, electricidad e incluso deportes. Asimismo, disfrutaban leer e investigar en estas áreas, pero tenían dificultades para involucrarse en diálogos con otras personas (p. e. pares, profesores) en cuanto al contenido de estas áreas de interés. Por último, en lo que respecta al ambiente de aprendizaje, se evidenciaba una necesidad exacerbada de previsibilidad, estructura y reglas, lo cual se traducía en tener ambientes lo más predecibles posible, con reglas y horarios claros y lógicos.

\section{Relación con profesores}

Los estudiantes con doble excepcionalidad habitualmente tienen dificultades en la relación con los profesores, principalmente por no ser capaces de cumplir las expectativas en torno a su desempeño académico, lo cual está asociado a críticas por no rendir de acuerdo a los estándares de los establecimientos (Bianco \& Leech, 2010).

Reis y Colbert (2004), en un estudio de estudiantes universitarios con doble excepcionalidad acerca de sus experiencias escolares, encontraron que muchas veces los profesores se sentían confundidos por las discrepancias manifestadas por los estudiantes en diferentes áreas. Los docentes también tendían a etiquetar a estos alumnos como "flojos" y que necesitaban "esforzarse más", y utilizaban diversas formas de castigo cuando los estudiantes no lograban completar alguna actividad, lo cual a su vez producía reticencias y episodios de rabia en ellos. Resultados similares fueron encontrados por Bredekamp (1993), quien reportó que la deserción en estudiantes doblemente excepcionales era producida por la falta de comprensión y apoyo por parte de profesores y personal de los establecimientos escolares.

Rubenstein y colaboradores (2015) encontraron que los estudiantes con TEA y alta capacidad presentaban dificultades a nivel escolar para responder en formas socialmente convencionales. Uno de los aspectos que afectaba la relación con los profesores era la poca capacidad que tenían los alumnos de responder adecuadamente a los gestos que estos 
tenían con ellos. Por ejemplo, cuando los profesores se esforzaban por ellos, los niños/as no podían expresar su gratitud o apreciación de la manera esperada.

Baum, Olenchack y Owen (1998) en un estudio de casos con estudiantes con TDAH y alta capacidad encontraron que los profesores habitualmente sancionaban a los estudiantes por responder impulsivamente en clases, sin respetar turnos. Además, tendían a interpretar su conducta de manera negativa, demostrando escasa comprensión de sus necesidades.

\section{Relaciones con pares}

Las relaciones entre pares son un aspecto muy relevante en la vida de una persona, el cual tiene diferentes expresiones, tales como la adaptación académica y social en la escuela (Patrick et al., 1999). Algunas investigaciones han reportado experiencias sociales poco satisfactorias en estudiantes con trastornos del aprendizaje. Para el caso de los estudiantes talentosos, la evidencia tiende a ser contradictoria: algunos han hallado un mal ajuste a nivel social, mientras que otros reportan relaciones positivas entre pares, especialmente con aquellos que tienen intereses similares (Lovecky, 2004). Sin embargo, dichos pares no siempre pueden ser encontrados en el ámbito escolar (Barber \& Mueller, 2011). Más aún, algunos estudios han reportado dificultades en los alumnos con doble excepcionalidad de congeniar o "encajar" con otros pares con alta capacidad, aquellos con trastorno del aprendizaje, o con otros pares en el aula regular (Nielsen, 2002).

En cuanto al trabajo en grupo dentro del aula, este ha sido considerado una buena experiencia para los estudiantes con doble excepcionalidad, por ejemplo, como una forma de expresar sentimientos en un grupo (Peterson, 2006). Willard-Holt y colaboradores (2013) encontraron que los estudiantes doblemente excepcionales tenían sentimientos ambivalentes acerca del trabajo en grupo y la colaboración con otros pares. Ello debido a que, si bien los estudiantes reconocían el beneficio de trabajar en grupos, algunos preferían actividades individuales porque querían asumir toda la responsabilidad o porque el 
trabajo grupal podía verse afectado por los problemas concomitantes a la doble excepcionalidad.

En cuanto a relaciones de amistad, Vespi y Yewchuk (1992) encontraron que los estudiantes con doble excepcionalidad podían tener dificultades o actuar de forma ambivalente con sus amigos(as). Aun cuando los estudiantes consideraran la amistad como un aspecto importante en sus vidas, mantener relaciones de amistad a través del tiempo era difícil de lograr. Asimismo, también estaban renuentes a acercarse a potenciales amigos debido al temor de ser rechazados. Por lo tanto, a pesar de tener habilidades para relacionarse socialmente, los estudiantes se sentían frustrados con las relaciones establecidas con sus pares y amigos.

Moon, Zentall, Grskovic, Hall y Stormont (2001) encontraron que, en los estudiantes con TDAH y alta capacidad, había una tendencia a ser emocional y socialmente más inmaduros que sus pares. También reportaron que, a nivel escolar, tendían a estar más aislados de sus pares pero eran capaces de encontrar amigos más allá de aquellos que tuvieran intereses y conductas similares. Sin embargo, otros investigadores como (Lovecky, 2004), encontraron que algunos estudiantes con alta capacidad y TDAH tenían un muy buen ajuste social, hacían amistades e incluso demostraban capacidad de liderazgo entre sus pares. No obstante, para los varones con TDAH el ámbito social se hacía un poco más dificultoso, con menos interacciones positivas entre pares.

En cuanto a estudiantes con TEA y alta capacidad, estudios más recientes sugieren que los sujetos son capaces de adaptarse mejor debido a una comprensión de las interacciones sociales, lo cual puede ser explicado en parte por su inteligencia verbal (Lovecky, 2004). Al comparar estudiantes con alta capacidad con y sin TEA, Neihart (2000) apuntó, entre otras cosas, a la falta de comprensión y dificultades que tienen quienes presentan ambas condiciones para seguir las reglas de la conducta social. En cuanto a las conductas y características de la interacción social, Foley Nicpon, Allmon, Sieck y Stinton (2011) encontraron que los padres de estudiantes con TEA reportaban conductas "extrańas" o atípicas en las interacciones con pares. 


\section{Ámbito socioafectivo y autopercepción}

Los rasgos socioemocionales de los estudiantes con doble excepcionalidad, al igual que cualquier individuo, son construidos a través de las relaciones establecidas con su contexto inmediato: familia, amigos y escuela. La diferencia, sin embargo, recae en los mensajes ambivalentes recibidos por estos estudiantes en relación con las percepciones que tienen las personas en su contexto sobre sus habilidades y dificultades, especialmente en el sistema escolar (Reis \& Colbert, 2004), lo cual puede traducirse en falta de confianza y baja autoeficacia en sus propias capacidades (Baum, Cooper \& Neu, 2001).

Se ha observado que los estudiantes doblemente excepcionales pueden tener un bajo autoconcepto académico debido a las dificultades que se presentan a nivel escolar. Esto es especialmente cierto en aquellos estudiantes cuyo potencial y habilidades están enmascarados por las dificultades asociadas a la doble excepcionalidad, lo cual puede derivar en baja autoeficacia y baja motivación hacia el éxito (Baum \& Owen, 1988) y resultar en conductas de agresión o aislamiento (Foley Nicpon et al., 2011). Las dificultades que presentan los estudiantes pueden llevarlos a creer que sus posibilidades de éxito son bajas, lo cual a su vez conlleva una sensación de desesperanza, baja motivación al logro y problemas con su autopercepción, sintiéndose diferentes y no tan capaces como sus pares (King, 2005; Vespi \& Yewchuk, 1992).

Vespi y Yewchuk (1992) en un estudio de casos fenomenológico, encontraron que los estudiantes con doble excepcionalidad, a pesar de experimentar diversas dificultades, tenían una visión positiva de sí mismos. Sabían que eran capaces y que tenían altas capacidades, y también tenían altas expectativas sobre su futuro. Sin embargo, cuando interactuaban con personas que tenían una mala percepción de sus habilidades, su autoimagen era menos positiva. En términos generales, tenían confianza en sus habilidades, pero al mismo tiempo se sentían responsables de sus fracasos.

Foley Nicpon, Rickels, Assouline y Richards (2012) midieron la autoestima y el autoconcepto en estudiantes talentosos con TDAH 
y sin TDAH. Encontraron que a pesar que ambos grupos tenían coeficientes intelectuales en rangos similares, los estudiantes con alta capacidad y TDAH tenían puntajes más bajos que sus pares talentosos en autoestima, autoconcepto y felicidad. Asimismo, encontraron que los estudiantes que habían sido identificados y rotulados como talentosos en sus establecimientos escolares y que tenían diagnóstico de TDAH también presentaban baja autoestima, sugiriendo que el rótulo de alta capacidad no tenía necesariamente un efecto positivo respecto a cómo el estudiante se sentía consigo mismo.

Un estudio similar fue realizado por Assouline, Foley Nicpon y Doobay (2009), en donde se compararon los perfiles de dos estudiantes talentosos con y sin TEA. Si bien no se observaron diferencias en cuanto al funcionamiento cognitivo y académico, sí se presentaban diferencias en sus autopercepciones: mientras la estudiante sin TEA reconocía dificultades sociales y emocionales, la estudiante con alta capacidad y TEA no reportó problemas en su autoconcepto. Según los autores, las respuestas positivas en escalas socioemocionales de autorreporte no son atípicas en este tipo de alumnos.

\section{El contexto chileno}

En el año 2009 en Chile se promulgó un importante decreto de ley que regula la atención a niños, niñas y jóvenes en educación especial. Este decreto número 170 está enfocado en proveer acceso igualitario y servicios para los estudiantes con necesidades educativas especiales en el sistema escolar regular en Chile, a través de programas especiales llamados Programas de Integración Escolar (PIE). Sin embargo, bajo esta ley, solo los estudiantes que presentan trastornos transitorios o permanentes están incluidos. No se hace referencia a una población igualmente vulnerable pero muchas veces ignorada: estudiantes con alta capacidad y estudiantes con doble excepcionalidad. Adicionalmente, la doble excepcionalidad es un área donde hay mucho desconocimiento en Chile y hasta la fecha no se han encontrado investigaciones sobre este tema. 
Sin embargo, esto no es lejano a lo que ocurre en otros países como Estados Unidos, en donde el debate acerca de proporcionar una atención adecuada a este grupo de estudiantes aún sigue en pie (Reis, Baum \& Burke, 2014).

El presente estudio tiene como propósito explorar las percepciones y experiencias de estudiantes chilenos con doble excepcionalidad con relación a sí mismos, y describir las relaciones que han construido en el contexto escolar. Las preguntas de investigación que guiaron el estudio fueron las siguientes: (a) ¿Qué experiencias han tenido los estudiantes con doble excepcionalidad en el contexto escolar?; (b) ¿Qué tipo de relaciones establecen?; y (c) ¿Cómo construyen sus autopercepciones como personas con doble excepcionalidad?

\section{Método}

Dada la escasez de investigaciones en Chile en esta temática, este estudio es considerado exploratorio y descriptivo, con el propósito de comenzar a generar conocimiento acerca de la doble excepcionalidad, identificando los elementos contextuales que influyen en este fenómeno y generando inferencias y explicaciones para investigaciones futuras (Ahonen, 2009). Debido a su naturaleza, se trata de un estudio cualitativo de casos múltiples, diseńado para explorar las semejanzas y diferencias entre casos (Stake, 2007). Es importante considerar que a partir de este diseño no siempre es posible extrapolar los resultados a la población general, sino que más bien se trata de inferencias clínicas, lo cual cumple con el objetivo de realizar una primera aproximación al fenómeno, y además permite generalizar entre los casos, para poder comprender su manifestación en profundidad (Geertz, 1987).

\section{Participantes}

La muestra estuvo compuesta por cuatro casos, todos ellos varones que viven en la región de Valparaíso, ubicada en la zona central de Chile. A continuación se entrega una breve descripción de cada uno de los casos: 
- S1 tiene 13 años y vive con ambos padres y cuatro hermanas mayores. Actualmente cursa séptimo básico en un colegio particular (privado) en donde ha cursado toda la enseñanza básica. Hasta quinto básico tuvo un buen rendimiento reflejado en sus notas, sin embargo hace dos años sus calificaciones bajaron, frente a lo cual los padres observaron una disminución en su motivación académica y bajo interés en tareas escolares. Fue evaluado fuera del colegio por especialistas, quienes diagnosticaron TDAH, para lo cual ha estado recibiendo apoyo semanalmente a través de intervenciones individuales.

- S2 tiene 15 años y vive con su madre, abuelos, una tía y su prima. Sus padres se divorciaron y su padre tiene dos hijos más. Durante su educación básica, S2 no tenía buenas notas, solo obtenía el mínimo para pasar de curso. En sexto básico su mamá buscó ayuda profesional para evaluarlo debido a su bajo rendimiento escolar, lo cual derivó en un diagnóstico de TDAH y medicación. En séptimo básico se cambió a un colegio particular y ha estado tratando de mejorar su rendimiento. Al momento de la investigación cursa primero de media.

- S3 tiene 14 años y vive con ambos padres y su hermana. Durante su educación básica tuvo un excelente rendimiento. Sin embargo, en séptimo básico tuvo que cambiarse de establecimiento debido a problemas socioemocionales resultantes de episodios de bullying en su colegio. Su diagnóstico actual es autismo de alto funcionamiento.

- S4 tiene 8 ańos y vive con su madre y abuela materna. Luego de repetir un año, cursa segundo básico por segunda vez en un colegio particular. Después de ser diagnosticado por un neurólogo con autismo de alto funcionamiento, asiste a sesiones individuales semanales en el centro de desarrollo cognitivo. 


\section{Medidas e instrumentos de medición}

El instrumento utilizado para identificar la doble excepcionalidad en la fase de screening fue el Test de Matrices Progresivas de Raven (Raven, Court \& Raven, 1993), escala general.

Para el estudio de casos, el principal instrumento fue una entrevista semiestructurada a profundidad, la cual fue construida sobre la base de sustentos teóricos de la literatura internacional sobre el tema de doble excepcionalidad. Un resumen de las preguntas de la entrevista se puede encontrar en el Anexo A. Las entrevistas tuvieron una duración aproximada de 30 minutos a una hora, y fueron realizadas en las dependencias de la Facultad de Educación de Pontificia Universidad Católica de Valparaíso, excepto una que se realizó en el establecimiento educacional del estudiante.

\section{Procedimiento y procesamiento de información}

Frente a la incipiente cantidad de información con respecto a los estudiantes con doble excepcionalidad en Chile, el muestreo para esta investigación fue tipo bola de nieve. Se contactó con un centro especializado al cual asisten niños, nińas y jóvenes de todas las edades para diagnóstico e intervención en las áreas neuropsicoeducativas. Con la finalidad de poder pesquisarlos, se implementó un proceso de capacitación a los profesionales de dicho centro en la temática de la doble excepcionalidad y posteriormente se discutieron los casos que fueran susceptibles de participar de la investigación. Todos los posibles casos ya habían sido diagnosticados previamente con TDAH o TEA a través de diferentes evaluaciones neuropsicológicas y el uso de criterios del DSM-5. Con los casos referidos para el estudio, se realizó una revisión a profundidad de fichas clínicas para determinar si cumplían con los criterios para ser considerados en este estudio.

Inicialmente, fueron preseleccionados ocho casos para formar parte de una evaluación con el propósito de identificar la alta capacidad. En esta ocasión, se les administró el Test de Matrices Progresivas de Raven (Raven, Court \& Raven, 1993). Después de esta aplicación, 
cinco casos que obtuvieron sobre el percentil 90 fueron identificados como doblemente excepcionales por presentar un diagnóstico dual; sin embargo, solo cuatro casos aceptaron continuar en el estudio.

Los estudiantes y sus padres accedieron a participar en la investigación mediante la firma de consentimientos y asentimientos informados. Los nombres aquí utilizados son ficticios.

Los datos obtenidos de las entrevistas fueron transcritos y luego se realizó un análisis en tres niveles: abierto, axial y selectivo (Strauss \& Corbin, 1990). Los datos fueron analizados a través de análisis de contenido cualitativo, lo cual implicó el desarrollo de un proceso de pre-análisis, identificación de conceptos y análisis de los segmentos de contenido, establecimiento de códigos y definiciones asociadas, integración de códigos, desarrollo de categorías, e integración final (Cáceres, 2003).

De igual manera, se realizó una triangulación de investigadores para asegurar la validez de los análisis. El equipo usó el software de análisis de datos cualitativos online Dedoose. Este tipo de software es utilizado ampliamente en el campo de la investigación cualitativa, y es una herramienta útil para organizar, explorar, integrar y reflexionar en torno a los datos obtenidos (Talanquer, 2014).

\section{Resultados}

Los resultados de esta investigación han sido divididos en dos ámbitos generales: escuela e individuo. Dentro de estos contextos, se analizan diversas categorías y sus correspondientes temas.

\section{Ámbito escolar}

El ámbito escolar ha sido definido como el contexto en donde ocurre la enseñanza y aprendizaje de los estudiantes con doble excepcionalidad, las formas en que abordan sus experiencias con sus cursos y contenido, y las relaciones que han establecido dentro de sus establecimientos escolares con pares y profesores. 
Aprendizaje. Las experiencias de aprendizaje a nivel escolar han sido variadas para los estudiantes que componen la muestra. Algunos de ellos son capaces de resolver problemas y responder preguntas antes que sus compañeros, y demuestran habilidades y motivación hacia el descubrimiento, involucrándose en áreas que son atractivas para ellos, como lo que ocurre con S2 (15 años, TDAH): "ehh, si, sobre todo en lo que es científico, como que tengo muchas facilidades con eso". Muchos de los estudiantes comparten expectativas similares acerca de sus experiencias de aprendizaje en los establecimientos. Si pudieran cambiar algo de sus colegios o clases, aumentarían el grado de libertad, flexibilidad y la oportunidad de contar con mayores experiencias prácticas: "que pudiera hacer lo que quiera [en clases]" (S4, 8 años, TEA); "sí, como por ejemplo en química, con un material más demostrativo" ( $S 1$, 13 años, TDAH). Uno de los estudiantes también expresó su deseo de tener actividades más desafiantes: "podría exigirnos un poquito más a nosotros para ser mejores o para entender mejor (S3, 14 años, TEA).

Todos los estudiantes han tenido algún tipo de dificultad con algún curso o contenido. La falta de habilidades a veces está estrechamente vinculada con una falta de motivación e intereses hacia la disciplina, como por ejemplo lo que le ocurre a S2 (15 años, TDAH) con su motricidad fina:

Porque no soy hábil para esas cosas, o sea música sí, pero encuentro que alguien te enseñe música, como que hace que la música se vuelva monótona, y repetitiva, y repetitiva, entonces eso aburre, porque yo en mi casa, o sea tengo un bajo y toco a veces, pero cuando quiero, no es como ya, música, no es como tan pasar materia, pero esas cosas [...], y de arte, no tengo nada de artista, nada [...], (risas), entonces como que nunca puedo hacer bien los trabajos, y todo, entonces, como que no me atrae, y tecnología [...] en realidad son entretenidas, son como proyectos que hacemos, pero, también como que, pierdo [...]

Por otra parte, algunos de los estudiantes tienen dificultades con ciertas habilidades, tales como escribir o en su motricidad gruesa, lo cual reduce su interés hacia temas específicos, así como sus percepciones 
de autoeficacia: "la que menos me gusta ir es a ciencias naturales [...] porque siempre tengo que escribir [...] (escribir) es una tortura” (S4, 8 años, TEA); "lo que me perjudica mucho es la educación física, es que yo no soy muy bueno y en muy exigente la educación física. Me gusta, pero es muy difícil" (S3, 14 años, TEA).

La motivación hacia el aprendizaje se refleja en la participación en clases que tienen los estudiantes, la cual aumenta cuando el contenido es de su interés: “(en clases de matemáticas) Bueno [...] sé que contesto mucho y siempre hago eso, pero es instinto. Siempre ha sido personalidad mía levantar la mano lo más posible (risa) digo no, responder de la forma más eficiente posible, no siempre logro eso, pero a veces" (S3, 14 años, TEA).

Las áreas de interés entre algunos de los participantes se relacionan con disciplinas científicas. S2 (15 años, TDAH), que está en enseñanza media (secundaria) se interesa por la física. A S1 le gusta matemática, especialmente porque percibe que tiene las habilidades necesarias para esta área:

Dos de los participantes también demuestran orientación al logro, especialmente porque han vivido situaciones en el pasado donde tuvieron que mejorar sus notas o su rendimiento escolar general: "Ehh, bueno ahí hacer clases, lo, hasta el año pasado era como, ya clases y no poner atención, pero ahora desde este año me he puesto las pilas, por lo del NEM [notas de enseñanza media] y todo eso. ehhh y bueno, poner atención, los apuntes [...]" (S2, TDAH, 15 ańos); "cuando me propongo hacer algo, siempre intento lograrlo" ( 1 1, 13 ańos, TDAH).

Relaciones. Se refiere a las relaciones que los estudiantes han construido dentro de sus establecimientos escolares, las cuales incluyen tanto a pares como profesores, y podrían ser tanto positivas como negativas, las que se presentan a continuación:

Pares. Los estudiantes tienden a valorar características positivas en sus pares y/o amigos, tales como el respeto, la amabilidad y la confianza. S4 (TEA), el participante más joven de la muestra, valora mucho a aquellos amigos que lo puedan defender, especialmente porque ha tenido dificultades con sus compańeros de curso y/o colegio. 
El respeto parece ser la cualidad más apreciada por S3 (14 años, TEA) en sus relaciones con pares: "pero por lo general me respetan y el respeto es la base para formar una amistad con alguien". Y la confianza es un elemento importante para S2 (15 años, TDAH): "creo que son ellos, son personas en los que de verdad creo, y puedo confiar y hablar, son cercanos". S1 e S3 también se relacionan con pares con los cuales pueden disfrutar: "son graciosos simpáticos [...] A veces muy hiperactivos algunos" (S1, 13 años, TDAH); "bueno, por ejemplo el Fernando, el Fernando es muy, es un chico muy gracioso" (S3, 14 años, TEA).

En términos de experiencias negativas con pares a nivel escolar, a muchos de los participantes no les gustan las interrupciones y que sus compañeros no pongan atención en clases:

Una cosa que no me gusta, en realidad nunca me ha gustado, son los que no hacen las cosas, que no ponen atención en clases, y todo eso, como que me molesta un poco, como, no sé si me enoja, pero es como "estay haciendo siempre estupideces, ponte a hacer algo", o que no hacen las tareas, sabían, tenían las cosas para hacerlas pero no lo hacen igual, o como que, simplemente lo hacen mal, como que $[\ldots]$ No $[\ldots]$ (S2, 15 años, TDAH).

Las experiencias relatadas son actuales o pasadas, algunas de las cuales se relacionan con vivencias de bullying por parte de compañeros de clase, como es el caso de S3 y S4, ambos con diagnóstico de TEA. En el caso de S4, esos episodios se traducen en sentimientos de rabia: "me dicen insultos, me andan molestando [...] (me dicen) tonto y después yo les quiero pegar con una pala. $Y$ dejarlos inconscientes, y luego los mando al centro de la tierra y los quemo" (S4, 8 años, TEA); "(el bullying que le hicieron a un amigo) solo era bullying psicológico, a mí me hicieron tanto físico y psicológico" (S3, 14 años, TEA)

En cuanto al trabajo grupal en el aula, algunos de los participantes reconocen la utilidad del trabajo en equipo para lograr ciertas metas, pero se sienten frustrados cuando sus pares no hacen el esfuerzo suficiente: "Encuentro que las cosas [en grupo] se pueden hacer más fáciles y más rápido" ( $\mathrm{S} 1,13$ años, TDAH); 
Doble excepcionalidad: análisis exploratorio de experiencias y autoimagen... / Gómez Arizaga et al.

En unas ocasiones, cuando tengo que hacer trabajos en grupo, mis compañeros no tienen... percepción de cuando uno debe trabajar y cuando no. Muchos de ellos cuando intento trabajar con ellos, suelen hablar. Suelen distraerse muy fácil y yo cuando quiero seguir nooo, gran parte no me hacen caso (S3, 14 ańos, TEA).

Profesores. La mayoría de las relaciones que los estudiantes han tenido con sus profesores tienen una connotación positiva: reconocen sus aspectos positivos, les demuestran respeto, y en algunos casos sienten afinidad con algunos profesores con los que comparten intereses similares:

Emmm, sí, me agradan, como que siempre le he tenido apego a esos profesores. Siempre estoy agradecido de que me enseñen [...] Me agrada el profesor de historia, por su personalidad y porque compartimos muchos gustos. Me agrada mi profesora de matemáticas, que es mi profesora jefe también, a veces le suelo dar problemas, porque a veces tengo problemas con un compańero, pero... usualmente me llevo bien con ella, me agrada (S3, 14 años, TEA).

Ehh..., bien. No hay ningún profesor con el que me lleve mal [...] bueno, es con la que más facilidades tengo, también que el profesor es súper simpático y también como de esto, de jugar en computador y todo, es como joven, entonces como que también se puede hacer chistes con él, cosas con él, entonces todo eso ha hecho que me ayude, que me guste aún más la física, y... me interesa, de hecho, me empezó a gustar la física por la serie The Big Ban Theory, que era como el físico, que todos.... "oh yo quiero", Sheldon... entonces como que lo veía siempre, y ojalá, entonces como que la física me ha gustado cada vez más (S2, 15 ańos, TDAH).

Solo S3 ha tenido experiencias negativas con profesores, más que nada porque no le agradaba la personalidad y comentarios inadecuados de uno de ellos:

Bueno hay más que uno que otro profesor que no me agrada del todo. Bueno, es que, por... que a veces no me gusta su personalidad, pero la mayoría me caen muy bien. [...] es como pesado, da comentarios incomodos, cuando no debería hacerlo (S3, 14 años, TEA). 


\section{Ámbito individual}

Este ámbito está relacionado con las diferentes formas en que los estudiantes de la muestra se ven y analizan a sí mismos como individuos, lo que está determinado por su autoimagen y autoconcepto.

Autoconcepto académico. Este tema se relaciona con cómo los estudiantes de la muestra se ven a sí mismos como individuos en el ámbito escolar. Esta imagen ha sido construida a partir de las relaciones establecidas con su ambiente académico. Muchos de los participantes tienen altas expectativas sobre su futuro y se ven a sí mismos obteniendo títulos profesionales.

Asimismo, algunos se perciben como líderes entre sus pares, dentro y fuera del aula: "en mi curso entre los hombres, como somos pocos, ehh, esos dos como que siempre me hacen caso, ya como que conseguimos que me hagan caso, entonces les digo "ya, haz esto", y va y lo hace" (S2, 15 años, TDAH); "soy el líder de mi club" (S4, 8 años, TEA).

El autoconcepto académico de los estudiantes se sustenta en la construcción de una imagen de sí mismos como personas con alta capacidad con dificultades. Muestran una clara comprensión de sus habilidades, como menciona S1 (13 años, TDAH) "Mmm soy curioso supongo, leo mucho a veces"; y S3 (14 años, TEA), quien se ve a sí mismo como poseedor de lo que él describe como un "sexto sentido": "bueno, sé que tengo un sexto sentido muy avanzado para mi edad, como lo comprobé en la anterior prueba que hice".

Los participantes también reconocen sus dificultades, a pesar de que no han sido informados formalmente sobre su condición de doble excepcionalidad. Asimismo, existe la creencia de que ciertas conductas pueden modificarse con el fin de adaptarse mejor a ciertas situaciones. Esto ocurre especialmente con S3 (14 ańos, TDAH), quien cree que puede mejorar sus interacciones sociales:

Que yo tengo un problema que se llama Síndrome de Asperger, me hace ser más solitario con, en comparación con los demás [...] Quiero salir del Asperger, digo no, no, no es posible curarse del Asperger, pero sin embargo es posible modificarlo y...mejorar mis habilidades sociales. 
Autoconcepto social. Los estudiantes tienen una visión crítica de cómo sus amigos los perciben, y se pueden encontrar diferencias entre los estudiantes de la muestra con TDAH y TEA. El primer grupo, S1 (13 años) y S2 (15 años), reconocen sus problemas como aspectos que pueden no gustar a sus amigos, especialmente su inconsistencia, como dice S2: puede que no les puede gustar mucho, como que, cosas así, cuando se vuelve muy repetida, soy muy no se inconsistente, como que dejo de ir, como que las empiezo a dejar de lado.

Por otra parte, los estudiantes con alta capacidad y TEA, mencionan que no tienen muchos amigos, pero realizan esfuerzos conscientes por mejorar esta situación, como lo describe S3 (14 años): no tengo muchos con quien hablar [...] Quizás es que a veces digo una más que otra curiosidad, que de vez en cuando no se me comportar, es que tengo problemas, he tenido problemas sociales por eso y quiero mejorarlo, quiero ser más amigo de alguien.

Finalmente, existe en S3 (14 años) una autopercepción de ser diferente de sus pares: "(me siento diferente) en mi forma de ver el mundo".

\section{Discusión}

En este estudio, brindamos una aproximación exploratoria al fenómeno de la doble excepcionalidad en Chile, la cual puede ser muy particular a los contextos en que se sitúan los participantes. Sin embargo, sienta las bases para futuras investigaciones en el área, y muchos de los resultados obtenidos se relacionan con lo que han encontrado otros investigadores en esta área. Si bien los diagnósticos eran diferentes, se pudieron encontrar muchos aspectos comunes para ambos grupos. Las principales diferencias aparecen en la interacción con pares, en donde los estudiantes con TEA tienden a sufrir una mayor discriminación en el ámbito escolar y muchas veces están más expuestos a episodios de bullying que sus pares con TDAH, dado que son menos comprendidos por otros en cuanto a sus conductas sociales, y han desarrollado un autoconcepto de "sentirse diferentes", focalizando sus esfuerzos en tratar de ser como sus pares y a veces hasta esconder su condición de otros. 
En cuanto al ámbito académico, los estudiantes parecen no enfrentar tantos desafíos como era lo esperado para el caso de la doble excepcionalidad y solo refieren a problemas menores que les ocurren (p. e. motricidad fina). Sin embargo, esto puede explicarse debido a que la mayoría de los estudiantes han recibido apoyo adicional por parte de especialistas por un largo período de tiempo y por lo tanto manifiestan sentimientos de autoeficacia en su desempeño escolar. Otra posible explicación es que las estrategias asociadas a su alta capacidad han compensado los problemas que emanan de las dificultades asociadas a su doble excepcionalidad, tal como ha sido descrito en la literatura (Willard-Holt et al., 2013).

De igual manera, su interés en lo académico parece estar mediado por la forma cómo las materias se ajustan a sus intereses, focalizando su atención y motivación en áreas que ellos disfrutan y en las cuales tienen las habilidades para tener éxito, como matemáticas y ciencias. Las actividades y/o contenidos presentados de forma monótona o repetitiva les hace sentirse menos involucrados y, por lo tanto, aburridos. Esto es consistente con lo que otros autores han encontrado acerca de la importancia de un ajuste adecuado entre actividades e intereses de los estudiantes, tal como lo notó Van Tassel-Baska y colaboradores (2009), sugiriendo que para los niños es importante que los profesores puedan motivarlos a mantener la atención hacia temas de interés. Cuando los estudiantes se sienten motivados, tienden a desafiarse más y ponen más esfuerzo en alcanzar las metas que se han propuesto. En este sentido, para los estudiantes de la muestra el profesor resulta una figura fundamental, especialmente aquellos con los cuales sienten una "conexión" debido a intereses disciplinares similares o características socioemocionales que promueven el involucramiento en tareas académicas.

Como con los profesores, las relaciones y la elección de pares es una parte importante en la vida de los estudiantes. Características como confianza, lealtad y acompañamiento ayudan a los estudiantes a navegar por su vida cotidiana y por los problemas que puedan surgir en sus establecimientos escolares. Las relaciones con pares en cuanto a tareas y trabajo grupal presenta mayores dificultades, dado que los 
estudiantes excepcionales en la muestra tienden a focalizarse en la tarea mientras que sus compańeros no necesariamente van a la par con ellos. Esto es consistente con lo encontrado por Willard-Holt y colaboradores (2013) en relación con la ambivalencia que manifestaban los alumnos doblemente excepcionales al trabajo en grupo: reconocen que es una parte importante de sus experiencias escolares pero prefieren trabajar solos.

Cuando se les pregunta sobre posibles cambios que les gustaría introducir para mejorar sus experiencias escolares, aparecen la necesidad de mayor desafío y flexibilidad como aspectos importantes del proceso de enseñanza y aprendizaje, así como también el incorporar mayores actividades de exploración, manipulación de materiales y aprender haciendo, aspectos que se ha encontrado permiten aproximar a los estudiantes doblemente excepcionales al aprendizaje (Silverman, 2003; Van tassel-Baska et al., 2009; Willard-Holt, 1998; Winebrenner, 2003).

El análisis del ámbito individual proporciona luces acerca de cómo los estudiantes con doble excepcionalidad en la muestra han construido su autoimagen basados en las interacciones con su contexto inmediato. Para este grupo, el aislamiento y las dificultades para hacer amigos es un aspecto importante. Sin embargo uno de los estudiantes activaba estrategias de compensación para enfrentar o resolver estas dificultades sociales, tales como hacer esfuerzos conscientes para acercarse y/o comunicarse con otros de la mejor forma posible. Esto se relaciona con la afirmación de Lovecky (2004) referida a que los estudiantes con alta capacidad y TEA son diferentes a los que solo tienen diagnóstico de TEA, dado que pueden comprender mejor las perspectivas sociales debido a habilidades verbales superiores que presentan.

Entre las limitaciones se observa que, dado el carácter cualitativo y exploratorio, la muestra fue limitada e incluyó estudiantes de establecimientos privados o semiprivados que podrían tener experiencias diferentes en relación con sus pares de establecimientos municipales. Esto podría constituir un sesgo dado que la educación chilena es socialmente estratificada y muy diversa. 
A pesar de ello, este estudio constituye un primer acercamiento al concepto de la doble excepcionalidad en Chile. Sin embargo, es necesario que se realicen investigaciones que incluyan no solo las percepciones de los estudiantes, sino también de otras figuras relevantes, como los padres, amigos y profesores, de modo tal que se pueda tener una visión más profunda y completa del concepto con el fin de analizarlo y poder tener una definición de cómo ocurre y se manifiesta en Chile.

Asimismo, este estudio puede ayudar a orientar el trabajo de profesionales y personal en las escuelas acerca de las principales características y dificultades que pueden presentarse en estudiantes con doble excepcionalidad, en este caso particular con diagnóstico de TDAH y TEA, con el propósito de entender sus conductas académicas y sociales, y poder brindar apoyo significativo a sus necesidades dentro y fuera del contexto escolar.

Para finalizar, se puede afirmar que los individuos en este estudio fueron capaces de delinear claramente un perfil de sí mismos como poseedores de características discrepantes, fortalezas y debilidades que son vistas de manera separada más que complementaria, lo cual sugiere que los estudiantes no siempre saben o están conscientes de su condición. No obstante, sí se sienten diferentes de sus pares en la forma en que se enfrentan y ven el mundo, a través de la ventana de la doble excepcionalidad.

\section{Referencias}

Ahonen, E., Porthe, V., Vazquez, M., Garcia, A., Lopez-Jacob, M., Ruiz-Frutos C. \& Benavides, F. (2009). A qualitative study about immigrant workers' perceptions of their working conditions in Spain. Journal of Epidemiology and Community Health, 63(11), 936-942. doi: 10.1136/jech.2008.077016

American Psychiatric Association [APA]. (2013). Guía de Consulta de los Criterios Diagnósticos del DSM-5 (A. A. d. Psiquiatría Ed.). Arlington, VA: American Psychiatric Publishing. 
Assouline, S. G., Foley Nicpon, M. F. \& Doobay, A. (2009). Profoundly gifted girls and autism spectrum disorder. Gifted Child Quaterly, 53, 89-105. http://dx.doi.org/10.1177/0016986208330565

Assouline, S. G., Foley Nicpon, M. F. \& Huber, D. (2006). The Impact of Vulnerabilities and Strengths on the Academic Experiences of Twice-Exceptional Students: A Message to School Counselors. Professional School Counseling, 10(1), 14-24. http://dx.doi. org/10.5330/prsc.10.1.y0677616t5j15511

Assouline, S. G. \& Whiteman, C. S. (2011). Twice-Exceptionality: Implications for School psychologists in the Post-IDEA 2004 Era. Journal of Applied School Psychology, 27(4), 380-402. http:// dx.doi.org/10.1080/15377903.2011.616576

Barber, C. \& Mueller, C. (2011). Social and Self-Perceptions of Adolescents Identified as Gifted, Learning Disabled, and TwiceExceptional. Roeper Review, 33, 109-120. http://dx.doi.org/10. 1080/02783193.2011.554158

Baum, S., Cooper, C. \& Neu, T. (2001). Dual differentiation: An approach for meeting the curricular needs of gifted students with learning disabilities. Psychology in the Schools, 38, 477-490. http://dx.doi.org/10.1002/pits. 1036

Baum, S. M., Olenchak, F. R. \& Owen, S. V. (1998). Gifted Students with Attention Deficits: Fact and/or Fiction? Or, Can We See the Forest for the Trees? Gifted Child Quarterly, 12(2), 96-104. http://dx.doi.org/10.1177/001698629804200204

Baum, S. \& Owen, S. (1988). High ability/learning disabled students: How are they different?. Gifted Child Quarterly, 32, 321-326. http://dx.doi.org/10.1177/001698628803200305

Baum, S. \& Owen, S. (2004). To be gifted and learning disabled: Strategies for helping bright students with $L D, A D H D$, and more. Mansfield Center, CT: Creative Learning Press.

BETA-PUCV (2015). Programa Educacional para Talentos Académicos. Sitio web. www.programabeta.cl

Bianco, M. \& Leech, N. (2010). Twice exceptional learners: effects of teacher preparation and disability labels on gifted referrals. 
Teacher education and special education, 33(4), 319-334. http:// dx.doi.org/10.1177/0888406409356392

Bredekamp, C. M. (1993). The Gifted/Learning Disabled Student: A Contradiction in the Classroom. Recuperado de http://files.eric. ed.gov/fulltext/ED374579.pdf

Cáceres, P. (2003). Análisis cualitativo de contenido: Una alternativa metodológica alcanzable. Psicoperspectivas, II, 53-82.

Dixon, F. \& Moon S. (2006). The Handbook of Secondary Gifted Education. Texas: Prufrock Press Inc.

Dole, S. (2000). The implications of the risk and resilience literature for gifted students with learning disabilities. Roeper Review, 23(2), 91-96. http://dx.doi.org/10.1080/02783190009554074

Flint, L. J. (2001). Challenges of identifying and serving gifted children with ADHD. Teaching Exceptional Children, 33(4), 62-69.

Foley Nicpon, M., Allmon, A., Sieck, B. \& Stinton, R. (2011). Empirical investigation of twice-exceptionality: Where have we been and where are we going? Gifted Child Quarterly, 55(3), 3-17. http://dx.doi.org/10.1177/0016986210382575

Foley Nicpon, M., Rickels, H., Assouline, S. G. \& Richards, A. (2012). Self-esteem and self-concept examination among gifted students with ADHD. Journal for the Education of the Gifted, 35(3), 220-240. http://dx.doi.org/10.1177/0162353212451735

Gagné, F. (2011). Academic Talent Development and the Equity Issue in Gifted Education. Talent Development \& Excelence, 3(1), 3-22.

Geertz, C. (1987). La interpretación de las culturas. Barcelona: Gedisa. Heller, K. A., Mönks, F. J., Sternberg, R. J. \& Subotnik, R. F. E. (2000). International Handbook of Giftedness and Talent. Oxford: Pergamon Press.

Hua, O., Shore, B. M. \& Makarova, E. (2012). Inquiry-based instruction within a community of practice for gifted-ADHD college students. Gifted Education International, 30(1), 74-86. http://dx.doi.org/10.1177/0261429412447709 
Kaufmann, F., Kalbfleisch, M. \& Castellanos, F. (2000). Attention deficit disorders and gifted students: What do we really know? NRC/GT Newsletter, Senior Scholars Series University of Connecticut.

King, E. (2005). Addressing the Social and Emotional Needs of TwiceExceptional Students. Teaching Exceptional Children, 38(1), 16-20. Lovecky, D. (2004). Different Minds. Gifted Children with AD/ HD, Asperger Syndrome, and Other Learning Deficits. United Kingdom: Jessica Kingsley Publishers Ltd.

Lubinski, D. (2004). Long-Term Effects of Educational Acceleration. In N. Colangelo, S. Assouline \& M. Gross (Eds.), A Nation Deceived: How Schools Hold Back America's Brightest Students. Volume II (pp. 23-38). Iowa City: The University of Iowa.

McCoach, D., Kehle, T., Bray, M. \& Siegle, D. (2001). Best Practices in the identification of gifted students with learning disabilities. Psychology in the Schools, 38(5), 403-411. http://dx.doi.org /10.1002/pits.1029

MINEDUC (2012). Estadísticas de la Educación 2012. Centro de Estudios MINEDUC. Santiago.

Moon, S. M., Zentall, S., Grskovic, J., Hall, A. \& Stormont, M. (2001). Emotional, social, and family characteristics of boys with $\mathrm{AD} /$ HD and giftedness: A comparative case study. Journal for the Education of the Gifted, 24, 207-247.

Neihart, M. (2000). Gifted Children With Asperger's Syndrome. Gifted Child Quarterly, 44(4), 222-230.

Neihart, M. (2003). Gifted Children with Attention Deficit Hyperactivity Disorder (ADHD). ERIC EC Digest \#E649. http://dx.doi.org/10.1177/001698620004400403

Neihart, M. (2008). Identifying and providing services to twice exceptional children. Handbook of Giftedness in Children (pp. 115-137). US: Springer.

Nielsen, M. E. (2002). Gifted students with learning disabilities: Recommendations for identification and programming. Exceptionality, 10(2), 93-111. http://dx.doi.org/10.1207/S153 27035EX1002_4 
Pardo de Santayana, R. (2002). Superdotación intelectual y trastorno por déficit de atención e hiperactividad (TDAHH). FAISCA Revista de Altas Capacidades, 9, 126-135.

Patrick, H., Ryan, A., Alfeld-Liro, C., Fredricks, J., Hruda, L. \& Eccles, J. (1999). Adolescents' Commitment to Developing Talent: The Role of Peers in Continuing Motivation for Sports and the Arts. Journal of Youth and Adolescense, 28(6), 741-763. http://dx.doi. org/10.1023/A:1021643718575

PENTA-UC (2015). Programa Educacional para Niños con Talentos Académicos. Recuperado de www.pentauc.cl

Pereles, D., Omdal, S. \& Baldwin, L. (2009). Response to Intervention and twice-exceptional learners: A Promising Fit. Gifted Child Today, 38(1), 8-15.

Peterson, J. (2006). Addressing Counseling Needs of Gifted Students. ASCA Professional School Counseling, 10(1), 43-51. http:// dx.doi.org/10.5330/prsc.10.1.b76h32717q632tqn

PROENTA-UFRO (2015). Programa Educacional para Niños con Talentos Académicos. Recuperado de www.proentaufro.cl

Raven, J. C., Court, J. H. \& Raven, J. (1993). Test de matrices progresivas. Escalas coloreada, general y avanzada. Manual. Buenos Aires: Paidós.

Reis, S. M., Baum, S. M. \& Burke, E. (2014). An Operational Definition of Twice-Exceptional Learners: Implications and Applications. Gifted Child Quarterly, 58(3), 217-230. http:// dx.doi.org/10.1177/0016986214534976

Reis, S. M. \& Colbert, R. (2004). Counseling Needs of Academically Talented Students with Learning Disabilities. Professional School Counseling, 8(2), 156-167.

Rinn, A. \& Reynolds, M. (2012). Overexcitabilities and ADHD in the Gifted: An Examination. Roeper Review, 34(1), 38-45. 10.1080/02783193.2012.627551. http://dx.doi.org/10.1080/0 2783193.2012 .627551

Rubenstein, L., Schelling, N., Wilczynski, S. \& Hooks, E. (2015). Lived experiences of parents of gifted students with autism spectrum 
disorder. The struggle to find appropriate educational experiences. Gifted Child Quarterly, 10.1177/0016986215592193. http:// dx.doi.org/10.1177/0016986215592193

Silverman, L. (2000). The Two-Edged Sword of Compensation: How the Gifted Cope with Learning Disabilities. In K. Kay (Ed.), Uniquely Gifted: Identifying and Meeting the Needs of Twice Exceptional Learners (pp. 153-165). Gilsum, NH: Avocus Publishing.

Silverman, L. (2003). Gifted Children with Learning Disabilities. In N. Colangelo \& G. A. D. (Eds.), The Handbook of Gifted Education, Third Edition (pp. 533-543). Boston: Allyn \& Bacon.

Stake, R. (2007). Investigación en Estudio de Casos. Madrid: Ediciones Morata.

Strauss, A. \& Corbin, J. M. (1990). Basics of qualitative research: Grounded theory procedures and techniques: Sage Publications, Inc.

Talanquer, V. (2014). Using Qualitative Analysis Software to Facilitate Qualitative Data Analysis. In D. Bunce (Ed.), Tools of Chemestry Education Research (pp. 83-95). Washington, DC: American Chemical Society. http://dx.doi.org/10.1021/bk-2014-1166. ch005

Tenorio, S. (2011). Formación Inicial docente y necesidades educativas especiales. Estudios Pedagógicos, 37(2), 249-265. http://dx.doi. org/10.4067/S0718-07052011000200015

Trail, B. (2006). Parenting Twice-Exceptional Children through Frustration to Success. Davidson Institute for Talent Development, 1-4. Recuperado de http://www.davidsongifted.org/db/Articles_ print_id_10462.aspx

Van Tassel-Baska, J., Feng, A., Swanson, J., Quek, C. \& Chandler, K. (2009). Academic and Affective Profiles of Low-Income, Minority, and Twice-Exceptional Gifted Learners: The Role of Gifted Program Membership in Enhancing Self. Journal of Advanced Academics, 20(4), 702-739. http://dx.doi.org/10.1177 /1932202X0902000406 
Vespi, L. \& Yewchuk, C. (1992). A Phenomenological Study of the Social/Emotional Characteristics of Gifted Learning Disabled Children. Journal for the Education of the Gifted, 16(1), 55-72.

Webb, J. \& Latimer, D. (1993). ADHD and children who are gifted. Reston, VA: Council for Exceptional Children

Willard-Holt, C. (1998). Academic and personality characteristics of gifted students with cerebral palsy: A multiple case study. Exceptional Children, 65(1), 37-50.

Willard-Holt, C., Weber, J., Morrison, K. \& Horgan, J. (2013). Twice-Exceptional Learners' Perspectives on Effective Learning Strategies. Gifted Child Quarterly, 57(4), 247-262. http://dx.doi. org/10.1177/0016986213501076

Winebrenner, S. (2003). Teaching Strategies for Twice-Exceptional Students. Intervention in School and Clinic, 38(3), 131-137. http://dx.doi.org/10.1177/10534512030380030101

Recibido el 25 de mayo de 2015 Aprobado el 30 de septiembre de 2015 


\section{Anexo A}

\section{Guía de la entrevista}

- Por favor cuéntame sobre ti, descríbete en tus aspectos positivos y negativos

- ¿Qué te gusta hacer?

1. Contexto escolar:

- ¿Te gusta tu escuela/colegio? ¿Cuáles son las cosas que te gustan y cuáles no te gustan de él? ¿Cómo te gustaría que fuera?

- ¿Te gusta ir a clases? ¿Por qué?

- ¿Cómo te sientes como estudiante? ¿Qué cosas parecen fáciles o más difíciles para ti?

- ¿Qué asignatura te gusta? ¿Y que asignatura no te gusta? ¿Por qué?

- ¿Cómo te llevas con tus profesores? ¿Por qué?

- ¿Participas en clases? ¿Cómo te gustaría que fueran tus clases?

- ¿Cómo te llevas con tus compañeros? ¿Qué cosas te gustan y que no te gustan sobre tus compañeros?

- ¿Cómo prefieres trabajar en clases? ¿Solo? ¿En grupo? ¿Por qué?

- ¿Si pudieras pedirle algo a tu colegio/escuela, qué seria?

\section{Relaciones sociales:}

- ¿Tienes amigos?

- ¿De dónde son tus amigos?

- ¿Cómo se hicieron amigos?

- ¿Cómo son tus amigos? ¿Qué cosas te gustan y que no te gusta de ellos?

- ¿Qué crees que a tus amigos les gusta de ti? ¿Qué crees que no les gusta?

- ¿Has tenido problemas con tus amigos? Si la respuesta es "si" preguntar: ¿Qué tipos de problemas? ¿Cómo resolvieron el problema

- ¿Sientes que eres diferente a tus amigos? ¿En qué área? O, ¿Crees que te ven de manera diferente? 